\section{Uso da autópsia verbal na investigação de óbitos com causa mal definida em Minas Gerais, Brasil}

\author{
Verbal autopsy for investigating deaths from \\ ill-defined causes in Minas Gerais State, Brazil
}

\begin{abstract}
Ill-defined causes of death can be related to problems in access to health services or poor quality of medical care and are indicators of data quality in the Mortality Information System (MIS). A sample of municipalities (counties) was selected from the Northeastern Macro-Region of Minas Gerais State, Brazil, with the aim of investigating deaths from ill-defined causes and deaths not reported to the Mortality Information System in 2007, using the verbal autopsy technique. The method allowed identifying 87\% of the causes of investigated deaths, of which $17 \%(n=37)$ were due to violent causes. At the end of the study, of the 779 investigated deaths, 9.5\% $(n=74)$ were due to external causes found outside the MIS. The distribution of causes was similar when comparing deaths reported (versus not reported) to the MIS for natural causes, but different when external causes were included. The article concludes that the verbal autopsy method can be a valuable tool for improving the MIS, allowing the identification of causes of death and improving data completeness.
\end{abstract}

Cause of Death; Mortality Registries; Information Systems
Deise Campos 1

Elisabeth França 2

Rosangela Helena Loschi 3

Maria de Fátima Marinho de Souza 4

As informações sobre causas de morte provenientes das estatísticas de saúde são de importância fundamental, pois fornecem subsídios para avaliar a situação de saúde das populações e para o planejamento, o monitoramento e a avaliação em saúde. As causas mal definidas de morte refletem a falta de acesso ao serviço de saúde ${ }^{1}$ e a qualidade da assistência médica. Podem ocorrer porque a pessoa chegou já falecida ao serviço de saúde ou porque houve omissão de informações por parte do atestante 2 . A proporção de óbitos com causas mal definidas é considerada um indicador para avaliar a qualidade de um sistema de informações sobre óbitos 3,4,5 e, quando elevada, os estudos das causas de morte, especialmente os estudos de séries temporais e as distribuições das causas por sexo e idade, podem ficar prejudicados.

Segundo avaliações internacionais, o Brasil apresentou elevada proporção de óbitos por causa mal definida ou inespecífica no período 1974-2000, e foi classificado como tendo qualidade "média" do seu sistema de informações sobre óbitos 3 . A cobertura e a qualidade das informações sobre causas de óbito são desiguais entre as regiões brasileiras e, apesar de importante decréscimo observado na proporção de óbitos por causa mal definida nos últimos anos, as regiões Norte e Nordeste ainda apresentam níveis muito elevados 5 . 
Para fazer frente a situações semelhantes a essa, em 2007, a Organização Mundial da Saúde (OMS) divulgou formulários padronizados para investigação de óbitos por meio do método da autópsia verbal nas áreas com elevada subnotificação e baixa cobertura da informação sobre causas da morte 6 . Trabalhos internacionais têm utilizado essa metodologia, especialmente em países onde é preciso lançar mão de metodologia específica para se conhecer a estrutura das causas de mortes para orientar políticas públicas em saúde. O método da autópsia verbal foi capaz de reduzir a proporção de mortes por causas inespecíficas e desconhecidas em adultos, tanto em áreas urbanas quanto rurais em um estudo que investigou 80 mil óbitos na Índia 7. Na China e na Etiópia, o método possibilitou a identificação de doenças crônicas e não-transmissíveis, dentre elas as causas violentas e doenças infecciosas 8,9,10. Estudos de validação sobre o método foram conduzidos em vários países 11,12,13,14.

No Brasil, a autópsia verbal está sendo adotada com um objetivo diferente: o de investigar mortes por causas mal definidas onde já existe um sistema de informações com cobertura nacional. Desde 2004, a Secretaria de Vigilância em Saúde do Ministério da Saúde desenvolve o Programa de Redução do Percentual de Óbitos com Causa Mal Definida nas regiões Norte e Nordeste do país com melhoria importante deste indicador. Em março de 2008, o Ministério da Saúde lançou um projeto para implantação da autópsia verbal no país como um método para investigação de óbitos por causas mal definidas. Os formulários da autópsia verbal da OMS 6 foram adaptados para as causas mais prevalentes no Brasil e sua análise poderá permitir ao médico identificar a seqüência de eventos que levou ao óbito 15 .

Minas Gerais, um dos 15 estados que participaram do projeto piloto nacional, apresenta alta proporção de óbitos com causa mal definida: foi de 14,2\% em 1997, com pequeno decréscimo em 2005 (11,5\%) (Rede Interagencial de Informações para a Saúde - RIPSA. Indicadores e dados básicos para a saúde. http://www.ripsa.org.br/php/ index.php, acessado em 21/Out/2008). Grandes diferenciais entre as 13 macrorregiões de saúde do estado podem ser observados (Ministério da Saúde. Informações em saúde. http://www. datasus.gov.br, acessado em 21/Out/2008). Em estudo que investigou as informações sobre nascimentos e óbitos coletados rotineiramente em Minas Gerais, observou-se que a Macrorregião Nordeste apresentou maior proporção de microrregiões e municípios considerados "não adequados" do ponto de vista da cobertura e da qualidade dos dados 16 .
Este trabalho tem como objetivo investigar as mortes por causas mal definidas e os óbitos não notificados ao Sistema de Informações sobre Mortalidade (SIM) na Macrorregião Nordeste de Minas Gerais, e verificar as possíveis causas desses óbitos de acordo com os diagnósticos definidos pelo método da autópsia verbal.

\section{Material e métodos}

Trata-se de uma pesquisa avaliativa sobre o SIM nos municípios da Macrorregião Nordeste de Minas Gerais, em 2007. Essa macrorregião constitui-se de 63 municípios distribuídos em oito microrregiões (Ministério da Saúde. Informações em saúde. http://www.datasus.gov.br, acessado em 31/Jan/2008). A proporção da população alfabetizada é a menor dentre as macrorregiões do estado. O município pólo, Teófilo Otoni, está localizado a $473 \mathrm{~km}$ de Belo Horizonte, capital do estado. A principal fonte de renda da macrorregião é a atividade agropecuária.

\section{Método de amostragem}

Para este estudo, as causas mal definidas são aquelas alocadas no Capítulo XVIII Sintomas, Sinais e Achados Anormais de Exames Clínicos e de Laboratório da 10a revisão da Classificação Internacional de Doenças (CID-10), códigos R00 a R99 17. Dos 4.878 óbitos ocorridos na região em 2007 (dados provisórios de abril de 2008 da Secretaria de Estado da Saúde de Minas Gerais SIM/SES), o capítulo das causas mal definidas representou 23\% $(n=1.124)$.

Para o cálculo do tamanho da amostra assumiu-se que a proporção a ser estimada seria de $80 \%$ de óbitos com causa bem definida (entre os óbitos com causa mal definida investigados), com margem de erro de 6\%, nível de confiança de $90 \%$ e correção para o tamanho da população e correção devida ao delineamento de 1,5 18,19. Estimou-se o tamanho da amostra em 197 óbitos mal definidos, incluídos $20 \%$ de perdas. A amostragem deu-se em três estágios, considerando cada município um conglomerado:

1) Os municípios foram ordenados e agrupados em estratos de acordo com a população estimada para 2007 (Ministério da Saúde. Informações em saúde. http://www.datasus.gov.br, acessado em 31/Jan/2008) em: (a) municípios de até 9.999 habitantes (49,2\%); (b) de 10.000 a 19.999 habitantes (31,8\%); e (c) com 20.000 habitantes ou mais (19\%);

2) Os conglomerados foram sorteados dentro de cada estrato, garantindo a mesma proporção de municípios na população e na amostra; e 
3) Em cada conglomerado sorteado, todos os óbitos ocorridos foram selecionados e se constituem nas unidades amostrais deste estudo. Os municípios selecionados foram: Águas Vermelhas, Campanário, Comercinho, Felisburgo, Frei Gaspar, Itaobim, Jequitinhonha, Machacalis, Serra dos Aimorés e Virgem da Lapa.

\section{Coleta de dados}

O estudo piloto foi conduzido em um dos municípios sorteados da amostra, para testar a logística de campo e os instrumentos da pesquisa.

\section{- Identificação das fontes de notificação e busca ativa de óbitos}

Foram inicialmente realizadas reuniões com os gestores locais (secretário de saúde e gerente de epidemiologia) para apresentar o projeto e para encaminhamentos às fontes de notificação de óbitos. Foram também realizadas reuniões com todas as Equipes de Saúde da Família do município para estimular a participação na pesquisa e enfatizar a importância das informações em saúde.

Realizou-se busca ativa de óbitos nas seguintes fontes: Serviço de Epidemiologia; Comitê de investigação de óbito materno, fetal e infantil; hospital; centros de saúde; informantes-chave; cartório; cemitério; setor da Prefeitura responsável pelos sepultamentos; e os quatro Postos de Medicina Legal (PML) da região.

Os meios para obtenção de informações foram o banco de dados do SIM do município; Declarações de Óbito (DO) arquivadas; as Fichas de Investigação de doenças de notificação compulsória e de óbitos; prontuários; livros de registro de óbito, internação e sepultamento; guia de sepultamento; Ficha D e informações verbais do enfermeiro do Programa Saúde da Família (PSF), agente comunitário de saúde (ACS) e zelador de cemitério. Em todos os municípios pesquisados, perguntou-se sobre a existência de parteiras assim como da ocorrência de nascimentos e óbitos neonatais domiciliares.

Os óbitos detectados na busca ativa foram comparados com a lista de óbitos do SIM/SES (abril de 2008). Para cada óbito encontrado não pertencente a essa lista foi aberta uma Ficha de Busca Ativa, onde foram anotadas variáveis de identificação e outras epidemiológicas (peso ao nascer e causas de morte).

\section{- Investigação dos óbitos detectados na busca ativa e por causas mal definidas}

Para todos os óbitos com causa mal definida e os encontrados na busca ativa de óbitos, utilizou-se uma Ficha de Investigação de Óbitos com Causa Mal Definida do Ministério da Saúde 15, adaptada para o projeto, para registro das informações verbais e de prontuários.

A investigação dos óbitos pelo método da autópsia verbal foi realizada por entrevistadores treinados. Os formulários da autópsia verbal são constituídos por uma série de blocos de questões sobre sintomas, sinais e circunstâncias da doença que levou à morte, doenças antecedentes e fatores de risco, atendimentos em serviços de saúde, resultados de exames, vacinação, antecedentes da mãe, da gestação e do parto no caso de crianças menores de um ano, e ainda informações que permitem identificar óbitos por causas maternas. Os formulários terminam com um quadro no qual o médico certificador registra as causas de óbito. Os formulários da autópsia verbal utilizados neste estudo foram três: (a) para crianças menores de 28 dias; (b) para crianças de 28 dias ou mais e menos de 10 anos de idade; e (c) para pessoas com 10 anos de idade ou mais 15 .

Foram entrevistados os familiares ou cuidadores da pessoa falecida nas seguintes situações: (a) óbitos detectados pela busca ativa sem emissão de DO, portanto não notificados ao SIM; (b) óbitos com emissão de DO (notificados ou não ao SIM), mas com a causa básica mal definida. Para facilitar a interlocução, um ACS da área de abrangência da residência dos entrevistados acompanhou o entrevistador, mas não participou da entrevista para garantir a confidencialidade. Em todos os casos foi utilizado um Termo de Consentimento Livre e Esclarecido.

Médicos generalistas treinados analisaram os formulários de autópsia verbal, as anotações de prontuário e as informações verbais colhidas nos municípios e definiram as causas de morte, as quais foram anotadas em formulário específico semelhante ao Bloco VI da DO. As causas foram codificadas e a causa básica foi selecionada segundo regras padronizadas da CID-10 por profissionais treinados.

Nos casos de óbitos confirmados de 2007 em que não foi possível estabelecer dia e mês do óbito, a data foi estipulada como sendo 1o de julho de 2007 para o cálculo da idade $(n=12)$, ou foram considerados com idade ignorada $(n=7)$ quando não havia data de nascimento. Cinco casos de sexo ignorado não foram esclarecidos porque a autópsia verbal não foi realizada. 


\section{Análise estatística}

Realizou-se a análise descritiva dos óbitos e das informações colhidas nas autópsias verbais. Calculou-se a subnotificação de óbitos em 2007 dividindo-se o número de óbitos não notificados ao SIM encontrados na busca ativa pela soma dos óbitos notificados ao SIM e dos óbitos não notificados localizados na busca ativa. As causas foram tabuladas segundo capítulos da CID-10 ou grupamentos específicos no caso das causas externas. As estimativas de proporção e o efeito do delineamento foram obtidos considerando o desenho de amostragem descrito anteriormente e utilizando-se a metodologia proposta na literatura 19. Para verificar se havia diferença entre as distribuições por causas entre o grupo dos óbitos notificados ao SIM e os investigados, utilizou-se o teste qui-quadrado com nível de significância de 0,05. Foram utilizados os programas Access, para entrada de dados, Excel versão 2007 (Microsoft Corp., Estados Unidos), para as análises descritivas, e Epi Info 6.04d (Centers for Disease Control and Prevention, Atlanta, Estados Unidos) e Minitab v. 14 (Minitab Inc., State College, Estados Unidos), para as análises estatísticas.

\section{Aspectos éticos}

O projeto foi aprovado pelo Comitê de Ética em Pesquisa da Universidade Federal de Minas Gerais, conforme o parecer no. ETIC 341/06. Foram cumpridos todos os princípios éticos que regem as pesquisas envolvendo seres humanos.

\section{Resultados}

O total de óbitos investigados nos municípios selecionados foi de 779 , sendo 573 notificados ao SIM/SES antes do início do trabalho de campo e 206 identificados na busca ativa (Figura 1). Portanto, observou-se uma subnotificação de 26,4\% dos óbitos, conforme a listagem do SIM/SES em abril de 2008.

Dentre os óbitos notificados ao SIM (Grupo A), 35,4\% eram mal definidos $(n=203)$. Dos óbitos não notificados ao SIM encontrados na busca ativa, 58,7\% tiveram a DO localizada (Grupo B), dos quais 26 eram por causa mal definida. Oitenta e cinco óbitos não notificados ao SIM não tiveram a DO localizada. Dos elegíveis para a realização da autópsia verbal foram excluídos 14 óbitos (um deles dentre os notificados ao SIM) cujos anos de falecimento eram diferentes de 2007 segundo a investigação domiciliar inicial. Ocorreram ainda 82 perdas principalmente porque os endereços de residência eram em áreas não cobertas pelo PSF ou em zona rural de difícil acesso. Dessa forma, realizaram-se 218 autópsias verbais, que resultaram em 189 óbitos com causa bem definida e 29 (13,3\%) com causa mal definida (Figura 1).

A distribuição dos 779 óbitos notificados e não notificados ao SIM/SES segundo grandes grupos de causas por sexo e faixa etária encontrase na Tabela 1. Dentre os óbitos não notificados ( $n=206)$, a maior parte era do sexo masculino e tinha 50 anos de idade ou mais. Observa-se uma concentração de óbitos por causa externa na faixa etária de 20 a 49 anos e no sexo masculino.

A Tabela 2 apresenta as respostas dadas pelos entrevistados durante a autópsia verbal a algumas questões selecionadas. A maior parte dos óbitos investigados por esse método ocorreu em domicílio, na zona urbana e recebeu tratamento em estabelecimento de saúde durante a doença que levou à morte. Observa-se um número elevado de casos em que o entrevistado afirma que a DO e (ou) a Certidão de Óbito não foi emitida. Vinte e oito entrevistados responderam que o óbito se deu por ferimento, acidente ou violência.

A Tabela 3 apresenta a distribuição dos óbitos investigados por autópsia verbal segundo causa, sexo e faixa etária. Foram mais freqüentes os óbitos por doenças cardiovasculares e causas externas em ambos os sexos.

A distribuição dos óbitos investigados neste estudo segundo capítulos da CID-10 está na Tabela 4. A primeira coluna se refere aos óbitos notificados ao SIM/SES antes do início do trabalho de campo (Grupo A), e a segunda aos óbitos não notificados ao SIM/SES cujas DO foram localizadas na busca ativa (Grupo B). Dos 218 casos investigados pelo método da autópsia verbal, 151 $(69,3 \%)$ corresponderam aos óbitos com causa mal definida notificados ao SIM/SES (Grupo C), 23 aos mal definidos não notificados com DO localizadas (Grupo D) e 44 aos óbitos não notificados cujas DO não foram localizadas na investigação (Grupo E). Os óbitos com causas definidas concentraram-se nos capítulos das doenças cardiovasculares, causas externas e neoplasias. Os por causa externa são apresentados segundo grupamentos mais detalhados de causas.

$\mathrm{O}$ efeito do delineamento estimado resultou em 1,392. Como se utilizou amostragem por conglomerado, esse valor acima de 1 era esperado e indica que o delineamento proposto está razoavelmente próximo do plano de amostragem aleatória simples.

As causas básicas mais freqüentes antes da investigação pela autópsia verbal foram morte sem assistência (R98) com 162 casos, e causas mal definidas (R99) com 45 casos. Após a investigação, as categorias mais freqüentes foram aci- 


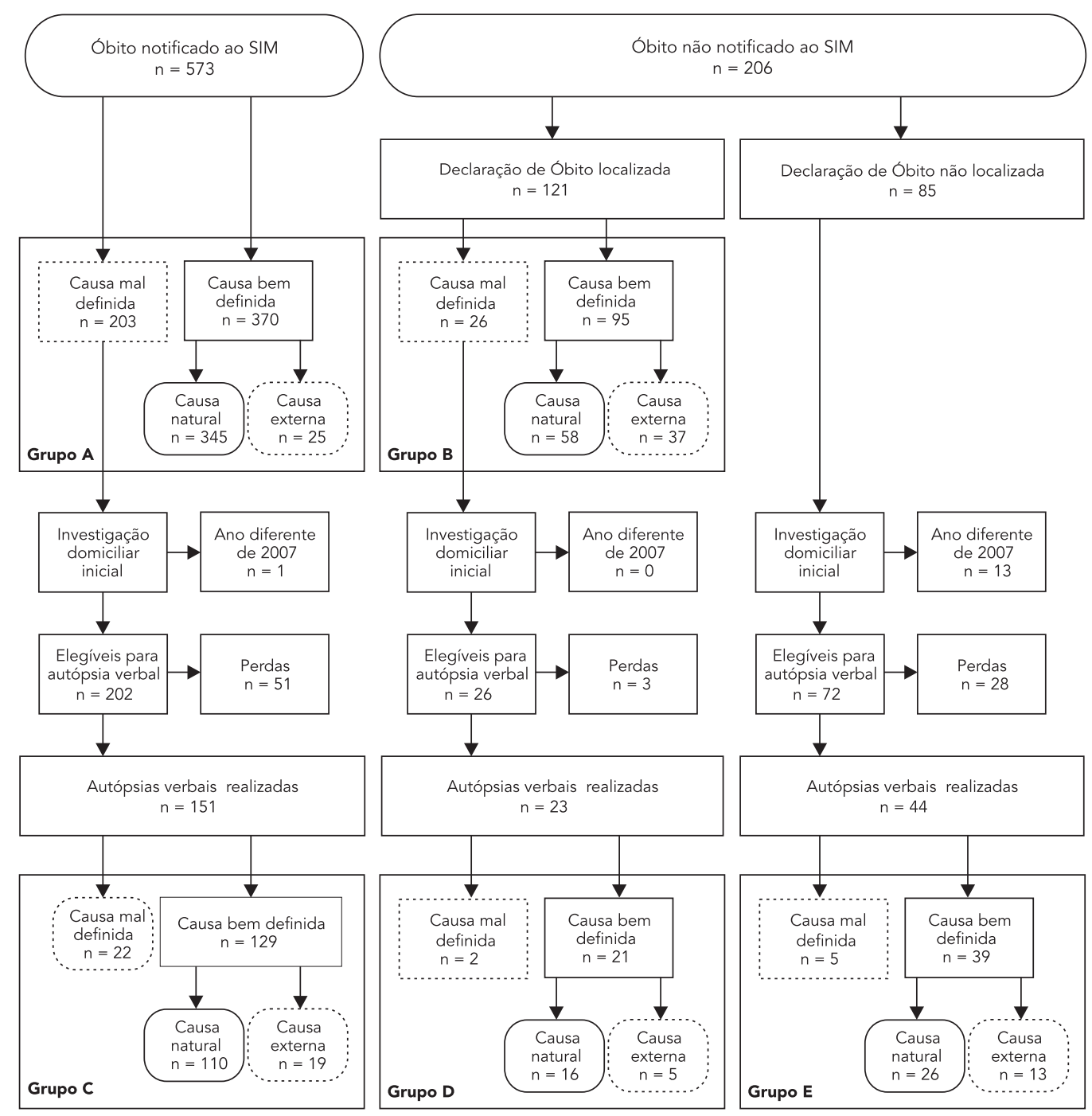

SIM: Sistema de Informações sobre Mortalidade.

dente vascular cerebral $(\mathrm{n}=68)$, infarto agudo do miocárdio $(n=32)$, hipertensão e doença cardíaca congestiva, todas do capítulo das doenças cardiovasculares. Nos demais capítulos da CID10, destacaram-se diabetes, pneumonia, doença pulmonar obstrutiva crônica, homicídio por arma de fogo, neoplasias (de esôfago, de estômago e de pulmão), septicemia, doença de Chagas, cirrose hepática alcoólica e alcoolismo (dados não apresentados).

Salienta-se que foram observadas 74 mortes por causas violentas não notificadas ao SIM/SES (Grupos B, C, D e E), das quais 28 foram devi- das a homicídio. Dentre os óbitos notificados ao SIM com causa mal definida e investigados pela autópsia verbal, 19 eram por causas externas. Dentre as autópsias verbais que resultaram em causas externas, chamou a atenção os entrevistados (familiares próximos) terem negado que os óbitos se deram por causa violenta em 11 casos (29,7\% das entrevistas), dos quais em sete a morte foi causada por queda. Conforme os relatos, em $59,5 \%$ dos casos $(n=22)$ a pessoa falecida fazia uso de bebida alcoólica e em 16,2\% ( $n=6)$ usava drogas. 
Distribuição dos óbitos segundo definição da causa básica e notificação ao Sistema de Informações sobre Mortalidade/Secretaria de Estado da Saúde de Minas Gerais (SIM/SES) por sexo e faixa etária. Macrorregião Nordeste de Minas Gerais, Brasil, 2007.

\begin{tabular}{|c|c|c|c|c|c|c|c|c|c|c|}
\hline \multirow[t]{2}{*}{ Causa da morte } & \multicolumn{8}{|c|}{ Faixa etária } & \multicolumn{2}{|c|}{ Total } \\
\hline & $\begin{array}{c}\text { Menor que } \\
28 \text { dias }\end{array}$ & $\begin{array}{l}28 \text { dias } \\
\text { a } 1 \text { ano }\end{array}$ & $\begin{array}{c}1-9 \\
\text { anos }\end{array}$ & $\begin{array}{l}\text { 10-19 } \\
\text { anos }\end{array}$ & $\begin{array}{l}20-49 \\
\text { anos }\end{array}$ & $\begin{array}{l}50-64 \\
\text { anos }\end{array}$ & $\begin{array}{c}65 \text { anos } \\
\text { e mais }\end{array}$ & Ignorada & $\mathbf{n}$ & $\%$ \\
\hline \multicolumn{11}{|l|}{ Óbitos notificados ao SIM/SES } \\
\hline Bem definidas & 12 & 12 & 3 & 3 & 72 & 60 & 208 & 0 & 370 & 64,57 \\
\hline Causas naturais & 12 & 12 & 1 & 1 & 59 & 54 & 206 & 0 & 345 & 60,21 \\
\hline Feminino & 4 & 3 & 0 & 1 & 25 & 21 & 100 & 0 & 154 & \\
\hline Masculino & 8 & 9 & 1 & 0 & 34 & 33 & 106 & 0 & 191 & \\
\hline Causas externas & 0 & 0 & 2 & 2 & 13 & 6 & 2 & 0 & 25 & 4,36 \\
\hline Feminino & 0 & 0 & 1 & 0 & 1 & 0 & 1 & 0 & 3 & \\
\hline Masculino & 0 & 0 & 1 & 2 & 12 & 6 & 1 & 0 & 22 & \\
\hline Mal definidas & 3 & 2 & 0 & 3 & 40 & 30 & 125 & 0 & 203 & 35,43 \\
\hline Feminino & 1 & 1 & 0 & 0 & 15 & 12 & 58 & 0 & 87 & \\
\hline Masculino & 2 & 1 & 0 & 3 & 25 & 18 & 67 & 0 & 116 & \\
\hline Total dos óbitos notificados & 15 & 14 & 3 & 6 & 112 & 90 & 333 & 0 & 573 & 100,00 \\
\hline \multicolumn{11}{|l|}{ Óbitos não notificados ao SIM/SES } \\
\hline \multicolumn{11}{|l|}{ localizados por busca ativa } \\
\hline Bem definidas (DO localizada) & 3 & 1 & 1 & 3 & 32 & 17 & 38 & 0 & 95 & 46,12 \\
\hline Causas naturais & 3 & 1 & 0 & 0 & 10 & 13 & 31 & 0 & 58 & 28,16 \\
\hline Feminino & 1 & 0 & 0 & 0 & 3 & 4 & 15 & 0 & 23 & \\
\hline Masculino & 2 & 1 & 0 & 0 & 6 & 9 & 16 & 0 & 34 & \\
\hline Ignorado & 0 & 0 & 0 & 0 & 1 & 0 & 0 & 0 & 1 & \\
\hline Causas externas & 0 & 0 & 1 & 3 & 22 & 4 & 7 & 0 & 37 & 17,96 \\
\hline Feminino & 0 & 0 & 0 & 0 & 4 & 1 & 1 & 0 & 6 & \\
\hline Masculino & 0 & 0 & 0 & 3 & 18 & 3 & 6 & 0 & 30 & \\
\hline Ignorado & 0 & 0 & 1 & 0 & 0 & 0 & 0 & 0 & 1 & \\
\hline Mal definidas (DO localizada) & 0 & 1 & 0 & 0 & 5 & 6 & 14 & 0 & 26 & 12,62 \\
\hline Feminino & 0 & 0 & 0 & 0 & 1 & 3 & 8 & 0 & 12 & \\
\hline Masculino & 0 & 1 & 0 & 0 & 4 & 3 & 6 & 0 & 14 & \\
\hline DO não localizada & 0 & 3 & 1 & 2 & 16 & 17 & 39 & 7 & 85 & 41,26 \\
\hline Feminino & 0 & 2 & 0 & 0 & 4 & 5 & 21 & 4 & 36 & \\
\hline Masculino & 0 & 1 & 1 & 2 & 11 & 11 & 18 & 2 & 46 & \\
\hline Ignorado & 0 & 0 & 0 & 0 & 1 & 1 & 0 & 1 & 3 & \\
\hline Total dos óbitos não notificados & 3 & 5 & 2 & 5 & 53 & 40 & 91 & 7 & 206 & 100,00 \\
\hline
\end{tabular}

DO: Declaração de Óbito.

Comparando-se a distribuição das causas naturais (causas bem definidas) entre os óbitos notificados ao SIM/SES (Grupo A - causas naturais) com a soma das demais colunas de causas naturais (Grupos B, C, D e E - causas naturais), encontrou-se forte evidência de que essas distribuições sejam semelhantes pelo teste do quiquadrado ( $p=0,237)$. Por outro lado, verificou-se forte evidência de desigualdade $(\mathrm{p}=0,000)$ quando comparou-se a distribuição do total das causas bem definidas dos óbitos notificados ao SIM/ SES (Grupo A - causas naturais e causas externas) com a soma das demais colunas, aí incluídas as causas externas (Grupos B, C, D e E - causas naturais e causas externas).

\section{Discussão}

Neste estudo, após a investigação, observou-se uma distribuição semelhante das causas naturais entre os óbitos notificados e não notificados ao SIM/SES, mas diferente quando incluídas as causas externas, com resultados consistentes quanto 
Respostas a questões selecionadas das autópsias verbais. Macrorregião Nordeste de Minas Gerais, Brasil, 2007.

\begin{tabular}{|c|c|c|}
\hline Variáveis selecionadas & $\mathrm{n}$ & $\%$ \\
\hline \multicolumn{3}{|l|}{ Local do óbito } \\
\hline Domicílio & 134 & 61,5 \\
\hline Hospital & 28 & 12,8 \\
\hline Via pública & 5 & 2,3 \\
\hline Outro & 51 & 23,4 \\
\hline \multicolumn{3}{|l|}{ Local da entrevista } \\
\hline Urbano & 173 & 79,4 \\
\hline Rural & 45 & 20,6 \\
\hline \multicolumn{3}{|l|}{ Relação do entrevistado com o(a) falecido(a) } \\
\hline Mãe ou pai & 15 & 6,9 \\
\hline Irmã ou irmão & 19 & 8,7 \\
\hline Cônjuge ou companheiro(a) & 45 & 20,6 \\
\hline Outro & 139 & 63,8 \\
\hline \multicolumn{3}{|l|}{ Por que não recebeu tratamento em serviço de saúde } \\
\hline Encontrou morto & 28 & 12,8 \\
\hline Não conseguiu chegar a tempo ao local & 20 & 9,2 \\
\hline Não quis/Não aceitou & 6 & 2,8 \\
\hline Não percebeu que precisava de tratamento & 6 & 2,8 \\
\hline Chegou ao local mas não teve atendimento & 3 & 1,4 \\
\hline Não se aplica (recebeu atendimento) & 140 & 64,2 \\
\hline Outro & 15 & 6,9 \\
\hline \multicolumn{3}{|l|}{ Outras questões * } \\
\hline Declaração de Óbito emitida & 126 & 57,8 \\
\hline Certidão de Óbito emitida pelo cartório & 164 & 75,2 \\
\hline Uso de bebida alcoólica & 92 & 42,2 \\
\hline Fumante & 104 & 47,7 \\
\hline Uso de drogas & 8 & 3,7 \\
\hline A pessoa faleceu devido a ferimento/acidente/violência & 28 & 12,8 \\
\hline Foi internado(a) durante a doença que levou à morte & 124 & 56,9 \\
\hline $\begin{array}{l}\text { Foi atendido(a) em outro estabelecimento de saúde durante a doença que levou à } \\
\text { morte (UBS, PSF, Clínica) }\end{array}$ & 103 & 47,2 \\
\hline Foi operado(a) por causa da doença & 22 & 10,1 \\
\hline
\end{tabular}

* Exclui "não sabe" e os que não quiseram responder.

à distribuição por sexo e faixa etária. Ou seja, a busca ativa e o método da autópsia verbal permitiram observar um novo perfil de mortalidade na Macrorregião Nordeste e identificar causas externas entre os óbitos notificados por causa mal definida, e também entre os não notificados ao SIM (17\% das entrevistas de autópsia verbal).

São poucos ainda os trabalhos brasileiros publicados que investigaram as causas mal definidas, buscando o seu esclarecimento por meio da busca de informações em prontuários, em visitas domiciliares ou por intermédio do pareamento com outras bases de dados. Em estudo realizado em 13 municípios dos estados de Sergipe, Mato
Grosso e São Paulo, alguns óbitos com causa mal definida foram realocados para o capítulo das causas externas. Os autores observaram ainda que a proporção de óbitos com causa mal definida que foi realocada para os outros capítulos da CID-10 foi menor entre as pessoas de 20 a 59 anos de idade, e a proporção por sexo foi diferente para cada capítulo da CID-10 20. De forma análoga aos resultados encontrados neste estudo, Rosa \& Garbin 21 verificaram que a maioria dos óbitos com causa mal definida era de pessoas portadoras de doenças crônicas em acompanhamento nos estabelecimentos de saúde e que faleceram no domicílio. Após investigação, os autores en- 
Tabela 3

Distribuição dos óbitos investigados por autópsia verbal segundo capítulo da Classificação Internacional de Doenças (CID), sexo e faixa etária. Macrorregião Nordeste de Minas Gerais, Brasil, 2007.

\begin{tabular}{|c|c|c|c|c|c|c|c|c|}
\hline \multirow{3}{*}{$\begin{array}{l}\text { Causa da morte após investigação pela } \\
\text { autópsia verbal }\end{array}$} & \multicolumn{8}{|c|}{ Feminino } \\
\hline & \multirow{2}{*}{$\begin{array}{c}28 \text { dias a } 1 \\
\text { ano }\end{array}$} & \multirow{2}{*}{\multicolumn{2}{|c|}{$\begin{array}{l}20-49 \\
\text { anos }\end{array}$}} & \multirow{2}{*}{$\begin{array}{l}50-64 \\
\text { anos }\end{array}$} & \multirow{2}{*}{$\begin{array}{l}65 \text { anos e } \\
\text { mais }\end{array}$} & \multicolumn{3}{|c|}{ Total } \\
\hline & & & & & & & n & $\%$ \\
\hline \multicolumn{9}{|l|}{ Bem definidas } \\
\hline \multicolumn{9}{|l|}{ Causas naturais } \\
\hline Doenças infecciosas & 0 & \multicolumn{2}{|c|}{2} & 2 & 2 & & 6 & 5,83 \\
\hline Neoplasias & 0 & \multicolumn{2}{|c|}{2} & 1 & 7 & & 10 & 9,71 \\
\hline Doenças dos órgãos hematopoiéticos & 0 & \multicolumn{2}{|c|}{0} & 0 & 0 & & 0 & 0,00 \\
\hline Doenças endócrino-metabólicas & 0 & \multicolumn{2}{|c|}{1} & 6 & 1 & & 8 & 7,77 \\
\hline Doenças mentais & 0 & \multicolumn{2}{|c|}{1} & 0 & 4 & & 5 & 4,85 \\
\hline Doenças do sistema nervoso & 0 & \multicolumn{2}{|c|}{0} & 1 & 1 & & 2 & 1,94 \\
\hline Doenças cardiovasculares & 0 & \multicolumn{2}{|c|}{0} & 6 & 29 & & 35 & 33,98 \\
\hline Doenças respiratórias & 0 & \multicolumn{2}{|c|}{0} & 0 & 5 & & 5 & 4,85 \\
\hline Doença do aparelho digestivo & 0 & \multicolumn{2}{|c|}{0} & 0 & 3 & & 3 & 2,91 \\
\hline Doenças do aparelho geniturinário & 0 & \multicolumn{2}{|c|}{0} & 0 & 0 & & 0 & 0,00 \\
\hline Causas perinatais & 0 & \multicolumn{2}{|c|}{0} & 0 & 0 & & 0 & 0,00 \\
\hline Causas externas & 1 & \multicolumn{2}{|c|}{5} & 1 & 8 & & 15 & 14,56 \\
\hline Mal definidas & 1 & \multicolumn{2}{|c|}{3} & 1 & 9 & & 14 & 13,59 \\
\hline Total & 2 & \multicolumn{2}{|c|}{14} & 18 & 69 & & 103 & 100,00 \\
\hline$\%$ & & & & & \multicolumn{4}{|c|}{47,25} \\
\hline \multirow{3}{*}{$\begin{array}{l}\text { Causa da morte após investigação pela } \\
\text { autópsia verbal }\end{array}$} & & & & & & & & \\
\hline & Menor que & 28 dias a & $10-19$ & $20-49$ & $50-64$ & 65 anos e & & \\
\hline & 28 dias & 1 ano & anos & anos & anos & mais & $\mathbf{n}$ & $\%$ \\
\hline Bem definidas & & & & & & & & \\
\hline Causas naturais & & & & & & & & \\
\hline Doenças infecciosas & 0 & 0 & 0 & 3 & 2 & 3 & 8 & 6,96 \\
\hline Neoplasias & 0 & 0 & 0 & 1 & 5 & 11 & 17 & 14,78 \\
\hline Doenças dos órgãos hematopoiéticos & 0 & 0 & 0 & 1 & 0 & 1 & 2 & 1,74 \\
\hline Doenças endócrino-metabólicas & 0 & 0 & 0 & 2 & 2 & 4 & 8 & 6,96 \\
\hline Doenças mentais & 0 & 0 & 0 & 0 & 0 & 1 & 1 & 0,87 \\
\hline Doenças do sistema nervoso & 0 & 0 & 0 & 3 & 0 & 2 & 5 & 4,35 \\
\hline Doenças cardiovasculares & 0 & 0 & 0 & 3 & 4 & 15 & 22 & 19,13 \\
\hline Doenças respiratórias & 0 & 0 & 0 & 0 & 1 & 7 & 8 & 6,96 \\
\hline Doença do aparelho digestivo & 0 & 0 & 0 & 2 & 2 & 1 & 5 & 4,35 \\
\hline Doenças do aparelho geniturinário & 0 & 0 & 0 & 0 & 1 & 0 & 1 & 0,87 \\
\hline Causas perinatais & 1 & 0 & 0 & 0 & 0 & 0 & 1 & 0,87 \\
\hline Causas externas & 0 & 0 & 3 & 10 & 5 & 4 & 22 & 19,13 \\
\hline Mal definidas & 1 & 1 & 0 & 4 & 0 & 9 & 15 & 13,04 \\
\hline Total & 2 & 1 & 3 & 29 & 22 & 58 & 115 & 100,00 \\
\hline$\%$ & & & & & & & 52,75 & \\
\hline
\end{tabular}

contraram uma maior migração de mortes para os capítulos das doenças cardiovasculares (40\%) e causas externas $(9,3 \%)^{21}$.

Estudos nacionais que avaliaram os dados registrados no SIM encontraram maior proporção de óbitos com causa mal definida em mulheres, pessoas de baixa escolaridade, cor da pele não branca, e entre aqueles ocorridos fora do hospital e por doenças crônicas ${ }^{2}$. Também Paes 22 encontrou correlação inversa entre a proporção de 
Distribuição dos óbitos segundo capítulo da Classificação Internacional de Doenças - 10ª revisão (CID-10), notificados, não notificados e investigados pela autópsia verbal. Macrorregião Nordeste de Minas Gerais, Brasil, 2007.

\begin{tabular}{|c|c|c|c|c|c|c|c|c|}
\hline \multirow[t]{4}{*}{ Causa da morte após a investigação } & \multirow{4}{*}{$\begin{array}{c}\text { óbitos } \\
\text { notificados } \\
\text { ao SIM/SES } \\
\text { Grupo A } \\
\text { n }\end{array}$} & \multirow{4}{*}{$\begin{array}{c}\text { óbitos não } \\
\text { notificados } \\
\text { ao SIM/ } \\
\text { SES (DO } \\
\text { localizada } \\
\text { na busca } \\
\text { ativa) } \\
\text { Grupo B } \\
\text { n }\end{array}$} & \multicolumn{6}{|c|}{ Investigados pela autópsia verbal } \\
\hline & & & \multirow{3}{*}{$\begin{array}{c}\text { óbitos com } \\
\text { causa mal } \\
\text { definida } \\
\text { notificados } \\
\text { ao SIM/SES } \\
\\
\text { Grupo C } \\
n\end{array}$} & \multirow{3}{*}{$\begin{array}{c}\text { óbitos com } \\
\text { causa mal } \\
\text { definida não } \\
\text { notificados ao } \\
\text { SIM/SES (DO } \\
\text { localizada na } \\
\text { busca ativa) } \\
\text { Grupo D } \\
\text { n }\end{array}$} & \multirow{3}{*}{$\begin{array}{c}\text { óbitos não } \\
\text { notificados } \\
\text { ao SIM/SES } \\
\text { (DO não } \\
\text { localizada } \\
\text { na busca } \\
\text { ativa) } \\
\text { Grupo E } \\
n\end{array}$} & \multicolumn{3}{|c|}{ Resultado da autópsia verbal * } \\
\hline & & & & & & \multicolumn{3}{|c|}{ Grupos C, D e E } \\
\hline & & & & & & $\mathrm{n}$ & $\%$ & IC95\% \\
\hline Total das bem definidas & 370 & 95 & 129 & 21 & 39 & 189 & - & - \\
\hline Causas naturais & 345 & 58 & 110 & 16 & 26 & 152 & - & - \\
\hline Doenças infecciosas & 30 & 4 & 9 & 1 & 4 & 14 & 6,01 & $2,29-9,74$ \\
\hline Neoplasias & 57 & 9 & 21 & 0 & 6 & 27 & 12,18 & $9,17-15,20$ \\
\hline Doenças endócrino-metabólicas & 23 & 5 & 12 & 3 & 1 & 16 & 6,751 & $3,32-10,18$ \\
\hline Doenças do sistema nervoso & 5 & 0 & 5 & 1 & 1 & 7 & 4,06 & $0,00-8,16$ \\
\hline Doenças cardiovascula-res & 132 & 22 & 38 & 8 & 11 & 57 & 27,69 & $19,28-36,10$ \\
\hline Doenças respiratórias & 36 & 1 & 11 & 1 & 1 & 13 & 6,17 & $1,56-10,79$ \\
\hline Doença do aparelho digestivo & 30 & 9 & 6 & 1 & 1 & 8 & 5,06 & $0,00-11,62$ \\
\hline Outras causas naturais $\star \star$ & 32 & 8 & 8 & 1 & 1 & 10 & 5,49 & $0,00-12,27$ \\
\hline Causas externas & 25 & 37 & 19 & 5 & 13 & 37 & 14,19 & $9,85-18,53$ \\
\hline $\begin{array}{l}\text { Acidente de transporte } \\
\text { especificado (V10-V88 e } \\
\text { V90-V98) }\end{array}$ & 6 & 0 & 0 & 0 & 0 & 0 & - & - \\
\hline $\begin{array}{l}\text { Acidente de transporte não } \\
\text { especificado (V89 e V99) }\end{array}$ & 2 & 11 & 1 & 0 & 0 & 1 & - & - \\
\hline $\begin{array}{l}\text { Acidente especificado } \\
\text { (W20-W64 e W75-X58) }\end{array}$ & 2 & 1 & 1 & 0 & 0 & 1 & - & - \\
\hline Afogamento (W65-W75) & 2 & 0 & 6 & 1 & 2 & 9 & - & - \\
\hline Atropelamento (V01-V09) & 2 & 1 & 0 & 0 & 1 & 1 & - & - \\
\hline $\begin{array}{l}\text { Homicídio (X85-X92, X96-X98, } \\
\text { Y00-Y09, Y35-Y35) }\end{array}$ & 2 & 2 & 2 & 1 & 0 & 3 & - & - \\
\hline $\begin{array}{l}\text { Homicídio por arma de fogo } \\
\text { (X93-X95) }\end{array}$ & 6 & 11 & 1 & 2 & 1 & 4 & - & - \\
\hline $\begin{array}{l}\text { Homicídio por instrumento } \\
\text { cortante (X99) }\end{array}$ & 2 & 6 & 1 & 0 & 1 & 2 & - & - \\
\hline Queda (W00-W19) & 1 & 3 & 4 & 0 & 7 & 11 & - & - \\
\hline $\begin{array}{l}\text { Intenção indeterminada } \\
\text { (Y10-Y34) }\end{array}$ & 0 & 1 & 3 & 1 & 1 & 5 & - & - \\
\hline Outras causas (Y40-Z99) & 0 & 1 & 0 & 0 & 0 & 0 & - & - \\
\hline Causas mal definidas & 203 & 26 & 22 & 2 & 5 & 29 & 12,40 & $8,24-16,55$ \\
\hline Total & 573 & 121 & 151 & 23 & 44 & 218 & & \\
\hline
\end{tabular}

DO: Declaração de óbito; IC95\%: intervalo de 95\% de confiança; SIM/SES: Sistema de Informações sobre Mortalidade/Secretaria de Estado da Saúde de Minas Gerais.

* As estimativas foram obtidas levando-se em conta a estratificação e o conglomerado município (unidade primária de amostragem) ${ }^{19}$. Efeito do desenho: 1,392 ;

** Outras causas naturais incluem: doenças dos órgãos hematopoiéticos, doenças mentais, doenças do aparelho geniturinário e causas perinatais. 
causas mal definidas e a proporção de óbitos por causas externas (para as idades de 10-64 anos), a cobertura dos óbitos e o grau de urbanização para os óbitos não registrados no SIM.

Vários estudos internacionais que utilizaram a metodologia da autópsia verbal também observaram mudança na estrutura das causas de morte, redução das causas mal definidas, identificação de causas externas, sendo as doenças mais freqüentes alocadas no capítulo das doenças do aparelho circulatório e das causas externas 7,8, resultados estes semelhantes aos encontrados neste estudo. Por outro lado, as doenças transmissíveis foram identificadas como o grupo mais freqüente de causas de morte por Lulu e Berhane 10 , em estudo com adultos residentes em área rural. Os autores utilizaram um algoritmo para definir a causa da morte com base no formulário aplicado e concluíram que este deve ser o método indicado em regiões onde não há médico 10 .

Embora não fosse o foco deste trabalho, como subproduto da pesquisa obteve-se algumas informações sobre a subnotificação de óbitos. O elevado nível de subnotificação de óbitos encontrado na Macrorregião Nordeste de Minas Gerais reflete as desigualdades socioeconômicas e a marginalização de grupos populacionais em Minas Gerais, conforme anteriormente observado 16. Dessa forma, mais uma vez a desigualdade socioeconômica se faz presente, tornando as causas externas invisíveis, escondendo-as entre as mal definidas, alterando artificialmente a distribuição das causas de morte e prejudicando a análise do perfil epidemiológico nessa macrorregião. No entanto, a cobertura dos óbitos parece estar melhorando. Entre 2001 e 2005, a população se manteve praticamente constante e o número de óbitos notificados ao SIM aumentou, assim como a cobertura por Equipes de Saúde da Família (Ministério da Saúde. Informações em saúde. http://www.datasus.gov.br, acessado em 21/Out/2008). Deve-se levar em conta também que a subnotificação observada deve diminuir até o fechamento dos dados estaduais referentes a 2007.

As dificuldades de acesso geográfico devem ser consideradas: a região estudada caracterizase por estradas ainda não pavimentadas e fazendas isoladas. Encontrou-se um expressivo número de óbitos não notificados ao SIM sem assistência médica na busca ativa realizada nos Livros de Registro de Óbito e DO emitidas por cartório. No entanto, nove dos municípios visitados tinham pelo menos um médico residente e o outro distava menos de 15 minutos de um pólo de microrregião, o que deveria ser suficiente para que nenhuma DO fosse emitida pelos cartórios, conforme preconizado pelo Ministério da Saú- de 23 . Acredita-se que investimentos conjuntos e continuados das sociedades médicas, do Conselho Regional de Medicina, das escolas médicas e do Estado serão muito eficazes na diminuição dos óbitos sem assistência médica e na melhoria da qualidade da assistência. Ressalte-se que está prevista a implantação de um Serviço de Verificação de Óbito para atender as regiões de Jequitinhonha, Leste e Nordeste de Minas Gerais 24. Os médicos legistas dos Postos de Medicina Legal da região receberam treinamento na Polícia Civil e iniciaram suas atividades no segundo semestre de 2007, exceto o de Teófilo Otoni que é mais antigo. Acredita-se que, após o período de implantação, com adequação da estrutura física e dos fluxos de documentos e de um treinamento complementar pela SES, esses serviços sejam capazes de captar maior número de óbitos. No entanto, ainda devem ser superadas as dificuldades de acesso geográfico.

Em algumas autópsias verbais que resultaram em causas externas, o entrevistado não identificava a causa que levou à morte como sendo ferimento, violência ou acidente, provavelmente porque o óbito não se deu imediatamente após o evento. Dessa forma, na definição da causa básica do óbito o médico certificador deve utilizar todas as informações disponíveis e não ficar restrito somente ao relato espontâneo dos cuidadores. Esse é um diferencial importante do método da autópsia verbal e deve-se chamar a atenção para que o médico esteja atento a todas as questões dos formulários à procura de pistas para esclarecer a correta seqüência de eventos que levou à morte. Isso é particularmente importante na investigação de óbitos de mulher em idade fértil, pois o formulário da autópsia verbal possui questões específicas para identificar causas maternas.

A maior limitação deste trabalho foi a impossibilidade de realizar a autópsia verbal onde não havia a presença do PSF ou ACS ou transporte para o entrevistador, o que aconteceu principalmente na zona rural. Além disso, foi utilizada uma listagem dos óbitos notificados ao SIM do estado em abril de 2008 e esta lista provisória constituise também em limitação do estudo. Entretanto esse procedimento foi necessário pois de outra forma a realização das entrevistas estaria muito prejudicada pelo viés de memória e pela possibilidade de mudança de endereço dos cuidadores da pessoa falecida. Ressalta-se que a Portaria $n^{o}$. 20 do Ministério da Saúde, de 3 de outubro de 200325 (substituída pela Portaria $n^{o}$. 116, de 11 de fevereiro de 2009 26), estabelecia que o prazo legal para a remessa de dados do ano anterior para o Ministério da Saúde finalizaria em 10 de abril. É de se esperar, devido ao intenso contato 
dos pesquisadores com a equipe municipal, que a notificação aumente nos municípios visitados.

Outra limitação se deve à realização da autópsia verbal em período superior ao recomendado pelo Ministério da Saúde, que é de no máximo três meses após o evento 15. Entretanto, a busca de informações em prontuários hospitalar e ambulatorial, além das informações verbais dos ACS, possibilitou que a análise pelos médicos certificadores contasse com mais dados que apenas os informados pelos cuidadores da pessoa falecida em grande parte dos casos.

Ressalta-se, por outro lado, que o método da autópsia verbal possibilitou esclarecer $87 \%$ das causas dos óbitos investigados. Além disso, outro ponto altamente positivo deste estudo foi o uso de instrumentos de autópsia verbal adaptados para o Brasil 15, pois tem sido recomendada a utilização de formulários padronizados, com cuidadosa tradução e adaptação da linguagem para diferentes regiões, tornando os resultados comparáveis internacionalmente 14. Por outro lado, apesar do método da autópsia verbal ser considerado uma estratégia importante para melhorar a qualidade das informações sobre mortalidade, é necessária sua validação segundo causas específicas de morte 13,14 .
Em resumo, conclui-se que a metodologia da autópsia verbal pode ser utilizada para esclarecer causas de morte, até que o SIM atinja níveis adequados de qualidade na definição da causa básica do óbito. Mesmo nos municípios que tradicionalmente possuem melhor qualidade da informação, o método pode ser utilizado pelo PSF como parte da metodologia de notificação e investigação de óbitos com causa mal definida. Concomitantemente, deve-se também trabalhar para aumentar a sensibilização dos médicos e acadêmicos de medicina quanto à importância de seu papel na produção de conhecimento sobre a situação de saúde da população.

Diversas secretarias de saúde do país têm procurado aprimorar o SIM de acordo com a realidade local 27,28. A melhor articulação entre os diversos atores desse sistema deve possibilitar que as questões relativas à qualidade das informações, à cobertura e ao fluxo de documentos sejam adequadamente enfrentadas, com o aprimoramento dos processos de trabalho. Espera-se assim, incentivar a incorporação sistemática de informações epidemiológicas confiáveis, necessárias para embasar a tomada de decisão segundo a lógica da assistência em pequenas localidades e nas Comissões Intergestoras Bipartites nas microrregiões de saúde.

\section{Resumo}

As causas mal definidas de morte refletem problemas de acesso aos serviços de saúde e de qualidade da assistência médica, e indicam precariedade no registro de dados no Sistema de Informações sobre Mortalidade (SIM). Selecionou-se uma amostra de municípios na Macrorregião Nordeste de Minas Gerais, Brasil, com o objetivo de investigar as mortes por causas mal definidas e os óbitos não notificados ao SIM em 2007, por meio do método da autópsia verbal. Esse método possibilitou esclarecer $87 \%$ das causas dos óbitos investigados, das quais $17 \%$ ( $n=37)$ eram por causas violentas. Ao final do estudo, dentre os 779 investigados, 9,5\% $(n=74)$ eram óbitos por causa externa encontrados fora do SIM. A distribuição por causas foi semelhante entre os óbitos notificados e não notificados ao SIM para as causas naturais, mas diferente quando incluídas as causas externas. Conclui-se que a investigação de óbitos com a metodologia da autópsia verbal pode ser um instrumento de grande valia para o aprimoramento do SIM no estado possibilitando o esclarecimento das causas de morte e também quanto à cobertura dos eventos.

Causas de Morte; Registros de Mortalidade; Sistemas de Informação; 


\section{Colaboradores}

D. Campos participou da concepção e delineamento do estudo, adaptação dos formulários da autópsia verbal, elaboração das fichas, elaboração dos manuais do entrevistador e da autópsia verbal, treinamento dos entrevistadores, levantamento bibliográfico, análise dos dados e redação final do artigo. E. França contribuiu com a concepção e delineamento do estudo, levantamento bibliográfico, análise dos dados e revisão crítica do artigo. R. H. Loschi participou da análise dos dados e revisão crítica do artigo. M. F. M. Souza contribuiu com o delineamento do estudo, adaptação dos formulários de autópsia verbal, análise dos dados e revisão crítica do artigo.

\section{Agradecimentos}

À Mariângela Cherchiglia e Daisy Abreu pelas sugestões. À Vera Barêa, Sandra Valongueiro e Bernadete Antunes que trabalharam na adaptação dos formulários de autópsia verbal para o Brasil. À Salete Diniz e Sueli Alvim da Secretaria de Estado de Saúde pelo apoio. À Ana Paula Travassos pelo cálculo do tamanho da amostra. À Priscila Kellen pela contribuição na busca ativa. Aos Bolsistas de Iniciação Científica Annelisa Lages, Lorena Machado e Pedro Trocoli. À Polícia Civil e secretarias municipais de saúde. Aos Entrevistadores e Equipes de Saúde da Família. À Fundação de Amparo à Pesquisa do Estado de Minas Gerais.

\section{Referências}

1. Organización Panamericana de la Salud. Sobre la estimación de tasas de mortalidad para países de la región de las Américas. Bol Epidemiol (Wash) 2003; 24:1-5.

2. Teixeira CLS, Klein CH, Bloch KV, Coeli CM. Reclassificação dos grupos de causas prováveis dos óbitos de causa mal definida, com base nas Autorizações de Internação Hospitalar no Sistema Único de Saúde, Estado do Rio de Janeiro, Brasil. Cad Saúde Pública 2006; 22:1315-24.

3. Matheus CD, Fat DM, Inoue M, Rao C, Lopez AD. Counting the dead and what they died from: an assessment of the global status of cause of death data. Bull World Health Organ 2005; 82:171-7.

4. Rao C, Lopez AD, Yang G, Begg S, Ma J. Evaluating national cause-of-death statistics: principles and application to the case of China. Bull World Health Organ 2005; 83:618-25.

5. França EB, Abreu DMX, Rao C, Lopez AD. Evaluation of cause-of-death statistics for Brazil, 20022004. Int J Epidemiol 2008; 37:891-901.

6. World Health Organization. Verbal autopsy standards: ascertaining and attributing cause of death. Geneva: World Health Organization; 2007.

7. Gajalakshmi V, Peto R. Verbal autopsy of 80.000 adult deaths in Tamilnadu, South India. BMC Public Health 2004; 4:47-54.

8. Joshi R, Cardona M, Iyengar S, Sukumar A, Raju $\mathrm{CR}$, Raju KR, et al. Chronic diseases now a leading cause of death in rural India: mortality data from the Andhra Pradesh Rural Health Initiative. Int J Epidemiol 2006; 35:1522-9.

9. Yang G, Rao C, Ma J, Wang L, Wan X, Dubrovsky G, et al. Validation of verbal autopsy procedures for adult in China. Int J Epidemiol 2006; 35:741-8.

10. Lulu K, Berhane Y. The use of simplified verbal autopsy in identifying causes of adult death in a predominantly rural population in Ethiopia. BMC Public Health 2005; 5:58-64. 
11. Ronsmans C, Vanneste AM, Chakraborty J, Ginneken JV. A comparison of three verbal autopsy methods to ascertain levels and causes of maternal deaths in Matlab, Bangladesh. Int J Epidemiol 1998; 27:660-6.

12. Anker M, Black RE, Coldham C, Kalter HD, Quigley MA, Ross D, et al. A standard verbal autopsy method for investigating causes os death in infants and children. Geneva: World Health Organization; 1999.

13. Chandramohan D, Setel P, Quigley M. Effect of misclassification of causes of death in verbal autopsy: can it be adjusted? Int J Epidemiol 2001; 30:509-14.

14. Setel PW, Rao C, Hemed Y, Whiting DR, Yang G, Chandramohan D, et al. Core verbal autopsy procedures with comparative validation results from two countries. PLoS Med 2006; 3:e268.

15. Departamento de Análise da Situação de Saúde, Secretaria de Vigilância em Saúde, Ministério da Saúde. Manual para investigação do óbito com causa mal definida. Brasília: Ministério da Saúde; 2008.

16. França E, Abreu D, Campos D, Rausch MC. Avaliação da qualidade da informação sobre mortalidade infantil em Minas Gerais, em 2000-2002: utilização de uma metodologia simplificada. Rev Méd Minas Gerais 2006; 16:S28-35.

17. Organização Mundial da Saúde. Classificação estatística internacional de doenças e problemas relacionados à saúde/CID-10. 8ạ Ed. São Paulo: Edusp; 2000.

18. Bolfarine HH, Bussab WO. Elementos de amostragem. Associação Brasileira de Estatística - Projeto Fisher. São Paulo: Editora Blucher; 2005.

19. Sousa MH, Silva NN. Estimativas de um levantamento complexo. Rev Saúde Pública 2003; 37: 662-70.

20. Mello-Jorge MHP, Gotlieb SLD, Laurenti R. O sistema de informações sobre mortalidade: problemas e propostas para seu o enfrentamento. I - mortes por causas naturais. Rev Bras Epidemiol 2002; 5: $197-211$
21. Rosa JAR, Garbin T. Redução das taxas de mortalidade por causas mal definidas em Bento Gonçalves (RS). In: Anais da 3a Expoepi - Mostra Nacional de Experiências Bem Sucedidas em Epidemiologia, Prevenção e Controle de Doenças. Brasília: Ministério da Saúde; 2003. p. 123-7.

22. Paes NA. Qualidade das estatísticas de óbitos por causas desconhecidas dos Estados Brasileiros. Rev Saúde Pública 2007; 41:436-45.

23. Ministério da Saúde. Manual de procedimentos do Sistema de Informações sobre Mortalidade. Brasília: Fundação Nacional de Saúde, Ministério da Saúde; 2001.

24. Minas Gerais. Resolução SES no ${ }^{\circ} .1 .318$, de 23 de outubro de 2007. Implanta a Rede Macrorregional de Serviços de Verificação de Óbitos do Estado de Minas Gerais. Minas Gerais 2007; 25 out.

25. Ministério da Saúde. Portaria $n^{\circ}$. 20, de 3 de outubro de 2003. Regulamenta a coleta de dados, fluxo e periodicidade das informações sobre óbitos e nascidos vivos para os Sistemas de Informação em Saúde - SIM e SINASC. Diário Oficial da União 2003; 9 out.

26. Ministério da Saúde. Portaria $n^{\circ}$. 116, de 11 de fevereiro de 2009. Regulamenta a coleta de dados, fluxo e periodicidade de envio das informações sobre óbitos e nascidos vivos para os Sistemas de Informações em Saúde sob gestão da Secretaria de Vigilância em Saúde. Diário Oficial da União 2009; 12 fev.

27. Simplício AMB, Iozzi R, Theme-Filha M, Caridade MC, Belizzi AL. Qualificação das bases de dados sobre mortalidade por causas violentas: recuperação das informações a partir do Sistema de Registro de Ocorrência da Polícia Civil na Cidade do Rio de Janeiro, 2001. In: Anais da 2a Expoepi - Mostra Nacional de Experiências Bem Sucedidas em Epidemiologia, Prevenção e Controle de Doenças. Brasília: Ministério da Saúde; 2002. p. 83-4.

28. Guimarães MJB. Aprimoramento do Sistema de Informações sobre Mortalidade: estratégias utilizadas pelo Município de Recife. In: Anais da 2a Expoepi - Mostra Nacional de Experiências Bem Sucedidas em Epidemiologia, Prevenção e Controle de Doenças. Brasília: Ministério da Saúde; 2002. p. 83-5.

Recebido em 13/Jan/2009

Versão final reapresentada em 10/Mar/2010

Aprovado em 05/Abr/2010 